CALET-

DOSCOPIO

23

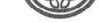

ENERO - JUNIO 2008

\title{
¿Cómo se relacionan los vocablos en un estudio de la disponibilidad léxica?
}

MARÍA GUADALUPE ROJAS CORONA/MARCO ANTONIO PÉREZ DURÁN

Benemérita Universidad Autónoma de Puebla Universidad Autónoma de San Luís Potosí

\section{INTRODUCCIÓN}

\section{$E_{1}$} análisis de la disponibilidad léxica ha tenido entre sus propósitos principales la obtención de los índices de disponibilidad de los vocablos que son utilizados en el discurso lingüístico en una comunidad específica. Esto se ha llevado a cabo en diversos tipos de investigaciones no solo en México sino a nivel mundial'. En cambio, no parece haberse intentado de manera suficiente describir y comparar al mismo tiempo cómo se relacionan los vocablos en un estudio de la disponibilidad léxica, cómo se estructuran estos vocablos en redes léxicas para formar constelaciones y cuáles son las relaciones asociativas que las rigen dentro del discurso lingüístico. Todo estudio de

En la actualidad se encuentra el proyecto Panhispánico sobre la disponibilidad léxica encabezado por Humberto López Morales. En México por su parte, los estudios sobre la disponibilidad léxica han sido estudiados por Juan López Chávez quien trabaja sobre índices de frecuencia de los vocablos para lograr una planificación lingüística. Asimismo en este país se ha conformado un grupo de estudiosos sobre el estudio de la disponibilidad, al cual nos incluimos, para generar estrategias que coadyuven a la mejora de la enseñanza de la lengua materna. 
la disponibilidad léxica se rige por centros de interés, los cuales son asociaciones o conjuntos de vocablos que denotan referencialidades específicas con la información solicitada del núcleo². Para este trabajo analizaremos el centro de interés "La Naturaleza", el cual forma parte de la clasificación tradicional, por lo menos en México, sobre el estudio de la variación y la disponibilidad del léxico de los informantes de una comunidad lingüística 3 .

Con este estudio se obtendrá: a) La selección de vocablos de acuerdo con los resultados léxico-estadísticos, b) La relación que los vocablos guardan en la muestra y c) Las asociaciones léxicas que se generan en el sistema lingüístico y que se verán reflejadas en la misma constelación. Este tipo de estudios se apoya en el léxico mental que engloba al léxico fundamental, básico, disponible y pasivo que se encuentran en la mente de los informantes y que se obtiene con base en colocar ciertos operadores léxicos para que el informante provea la mayor cantidad de respuestas para dicho núcleo, con ello pues se obtendrán las variaciones en primera instancia léxicas-estadísticas y en segundo lugar, las asociaciones léxicas-semánticas que cualquier vocablo engloba dentro de un discurso lingüístico. Tanto las variaciones estadísticas como semánticas del vocablo se ubican en la red léxica y en la constelación semántica, una perteneciente al habla y la otra a la lengua.

2 Marco Pérez, Investigación sobre la disponibilidad léxica del profesorado de secundaria del área de español, Tesis no publicada para optar al grado de Maestro en Lingüística Hispánica, México, UNAM, 2004, p. 56.

3 En México, los centros de interés para el estudio sobre la disponibilidad léxica son: 1) Partes del cuerpo, 2) La ropa, el vestido y el calzado, 3) La casa: el interior y sus partes, 4) Muebles y enseres domésticos, 5) Alimentos y bebidas, 6) Objetos colocados sobre la mesa, 7) La cocina y los utensilios, 8) La escuela, muebles y útiles, 9) Electricidad y aire acondicionado, 10) La ciudad, 11) La naturaleza, 12) Medios de transporte, 13) Trabajo de campo y jardinería, 14) Animales, 15) Diversión y deportes y 16) Oficios y profesiones. 


\section{1) Marco Teórico}

En México, los estudios de disponibilidad léxica cuya base sea la léxico-estadística y la léxico-semántica, se han ido incrementando conforme ha pasado el tiempo. Todos estos estudios parten de obtener un listado de vocablos con un índice de disponibilidad confiable que determine el grado de aparición y frecuencia de un vocablo en el código lingüístico y su relación con la referencialidad, la base de la cual se parte para el estudio léxico estadístico es el centro de interés, y la base de la que se parte para el estudio de la léxico-semántica es el centro nocional.

Un centro de interés es un campo de información referencial específico que se ubica en la lengua, de este centro se genera una relación de información para acrecentar el conocimiento y el dominio de la lengua a través de índices comparativos de respuestas que van apareciendo en el mismo centro ${ }^{4}$; o bien, son estímulos, los cuales, permiten a los informantes llevar a cabo un proceso de recuperación para traer a la luz las palabras que en su mente están relacionadas con un tema específico ${ }^{5}$.

Todo centro de interés es un núcleo mayor de información, por ello, se le conoce -también - como centros nocionales, semánticos, contextuales; etcétera ${ }^{6}$. Aunque Ullman ${ }^{7}$ separa de manera tajante la diferencia entre el centro de interés y el centro nocional. Para él, un centro de interés estará abocado única y exclusivamente a la obtención de léxico de un informante, en tanto que un centro nocional o

4 Pérez, op. cit., p. 102.

5 Juan López Chàvez, ¿Qué te viene a la memoria? La disponibilidad léxica: teoría, métodos y aplicaciones, México, Universidad Autónoma de Zacatecas, 2004, p. 256.

6 Meter Johnston, La evaluación de la comprensión lectora. Un enfoque cognitivo, Madrid, Visor, 1998, p. 45.

7 Stephen Ullman, Semántica, Introducción a la Ciencia del Significado, Madrid, Aguilar, 1978, p. 66. 
campo semántico es un grupo de vocablos obtenidos de un corpus a partir de las relaciones que se generan tanto en su significado como en su estructura; por tal motivo, un centro nocional es más complejo porque denota no sólo las características de estructuración sino de significación ${ }^{8}$.

Un centro de interés se enfocará más al léxico porque genera una relación estadística precisa con la cantidad de respuestas que surgen de un informante y es indispensable proveerlas de un índice de disponibilidad; en tanto que, un campo nocional o semántico reflejará las particularidades del significado de esos vocablos con relación a su distribución significativa en el discurso. Ambos parten del léxico, uno es más gramatical -centro de interés- y el otro es más significativo - centro nocional-.

La unidad mínima del centro de interés es el vocablo, mientras que la unidad mínima del centro nocional o semántico es la palabra9. Ambas son indispensables en la lexicología porque se agrupan en conjuntos de listemas. Un listema es una asociación de vocablos y de palabras que se fundamentan en el léxico, el cual es el conjunto de palabras de una lengua, o bien, el conjunto de palabras pertenecientes a una región determinada. ${ }^{10}$

Este léxico, a su vez, se agrupa en un léxico mental. Éste es el conjunto de todas las palabras que un hablante posee de su lengua materna y que utiliza como registro específico en su código lingüístico ${ }^{11}$.

8 El centro nocional se codifica en el sistema lingüístico, en tanto que el centro de interés se da en la norma. Ya que el primero es una designación del significado del vocablo que se verifica en su distribución con otros vocablos, en tanto que el centro de interés se da como parte del repertorio lingüístico que cualquier hablante posee de su lengua, agrupado en un conjunto, el cual está íntimamente ligado con los vocablos más representativos de la muestra.

9 Saussure (1916) define a la palabra y al vocablo como dos unidades distintas, la primera pertenecerá a la semántica mientras que la segunda a la morfología; una estará en el sistema y la otra en la norma. Una será el significado general que denota en la lengua mientras que el vocablo estará establecido por los rasgos de composición que se generan al interior del mismo.

10 López Chávez, op. cit., 2004, p. 332.

11 André Martinet, Elementos de Lingüistica General, Madrid, Gredos, 1966, p. 34. 
Este léxico mental se constituye por un léxico básico, disponible, fundamental y pasivo. El primero es el que un individuo posee como necesario en su discurso lingüístico, el segundo es el que habitualmente un hablante posee de su lengua para un momento determinado; el tercero es el léxico que debe tener un hablante como registro de su lengua y el léxico pasivo es el conjunto de vocablos que se ubican en la mente del individuo, no aparecen sino cuando son requeridos en una conversación. Asimismo, el léxico mental se desarrolla al interior de las asociaciones que requerimos en la lengua y en una conversación, se da según Saussure ${ }^{12}$ en el eje paradigmático, porque permite la elección de diferentes entradas léxicas a un código lingüístico.

El discurso lingüístico necesariamente partirá de las relaciones entre el léxico requerido para ejemplificar un proceso comunicativo coherente, por ello, el hablante tiene la capacidad lingüística de elegir el vocablo correcto para una situación específica. Esta selección del léxico se rige a partir unidades de selección denominadas: "operadores léxicos". Estos operadores son vocablos que activan a otros vocablos en un conjunto de asociaciones léxicas que se reflejan en el discurso; determinan relaciones de taxonomía en la lengua ${ }^{13}$. Un operador léxico constituye bases que especifican asociaciones directas con respecto al corpus. Esta asociación comprende mundos conceptuales específicos que se ubican en relaciones al léxico, o bien, a entidades subjetivas que denotan grados de referencialidad ${ }^{14}$. Una red léxica es -entonces- un sistema que está integrado por vocablos con índices de respuestas diferentes y que conforman en conjunto, una gran relación asociativa-significativa con la totalidad de las constelaciones que se rigen por asociaciones dentro de la lengua y dentro del léxico ${ }^{15}$.

Ferdinad de Saussure, Curso de Lingüística General, México, Nuevomar, 1916.

Ullman, op. cit., p. 57.

Pérez, op. cit., p. 104.

Saussure, op. cit., p. 124.

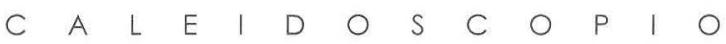




\section{2) Metodología}

Este trabajo de investigación originalmente forma parte de un proyecto de investigación mayor intitulado "Variación léxica en docentes de secundaria del estado de Tlaxcala". El cual parte del análisis de 16 centros de interés en un corpus de 136 informantes que participaron para dicha investigación. En este trabajo se analizaron 4 variables: 'género', 'nivel educativo', 'subsistema escolar' y 'edad'; con lo cual se obtuvo 36, 177 palabras como respuestas para el análisis general. Para este trabajo se tomó la muestra depurada que corresponde a 280 palabras, de éstas se obtuvieron los vocablos más disponibles de la muestra, cuyo resultado es de 38 vocablos. Tanto el primer resultado -280 cómo el segundo -38 - se tomarán para los análisis léxico-estadístico y léxico-semántico.

\subsection{Análisis Léxico-estadístico}

El centro que tomo para este trabajo es el centro "la naturaleza" A continuación se expone el cuadro de respuestas.

Cuadro 1: Apariciones y Porcentajes de los vocablos de este Centro

\begin{tabular}{|c|c|c|c|c|}
\hline $\begin{array}{l}\text { Número de } \\
\text { vocablos }\end{array}$ & Vocablos & $\begin{array}{c}\text { Número de } \\
\text { repeticiones en la } \\
\text { muestra }\end{array}$ & Porcentaje & Grupo \\
\hline 7 vocablos & $\begin{array}{l}\text { 1.-Agua } \\
\text { 2.-Aire } \\
\text { 3.-Árbol } \\
\text { 4.-Mar } \\
\text { 5.-Flor } \\
\text { 6.-Montaña } \\
\text { 7.-Río }\end{array}$ & 14 & $35 \%$ & Grupo 1 \\
\hline 4 vocablos & $\begin{array}{l}\text { 1.-Nube } \\
\text { 2.-Lluvia } \\
\text { 3.-Bosque } \\
\text { 4.-Sol }\end{array}$ & $\begin{array}{c}13 \\
\text { (52 repeticiones) }\end{array}$ & $18.57 \%$ & Grupo 2 \\
\hline 2 vocablos & $\begin{array}{l}\text { 1.-Animal } \\
\text { 2.-Planta }\end{array}$ & $\begin{array}{c}12 \\
\text { (24 repeticiones) }\end{array}$ & $8.57 \%$ & Grupo 3 \\
\hline 2 vocablos & $\begin{array}{l}\text { 1.- Pasto } \\
\text { 2.-Volcán }\end{array}$ & $\begin{array}{c}11 \\
\text { (22 repeticiones) }\end{array}$ & $7.85 \%$ & Grupo 4 \\
\hline 2 vocablos & $\begin{array}{l}\text { 1.-Tierra } \\
\text { 2.-Lago }\end{array}$ & $\begin{array}{c}10 \\
\text { (20 repeticiones) }\end{array}$ & $7.14 \%$ & Grupo 5 \\
\hline 1 vocablo & 1.-Laguna & 9 & $3.21 \%$ & Grupo 6 \\
\hline 2 vocablos & $\begin{array}{l}\text { 1.-Cielo } \\
\text { 2.-Pájaro }\end{array}$ & $\begin{array}{c}6 \\
\text { (12 repeticiones) }\end{array}$ & $4.28 \%$ & Grupo 7 \\
\hline
\end{tabular}




\begin{tabular}{|c|c|c|c|c|}
\hline 1 vocablo & 1.-Fauna & 5 & $1.78 \%$ & Grupo 8 \\
\hline 3 vocablos & $\begin{array}{l}\text { 1.-Calor } \\
\text { 2. Cerro } \\
\text { 3.-Viento }\end{array}$ & $\begin{array}{c}4 \\
\text { (12 repeticiones) }\end{array}$ & $4.28 \%$ & Grupo 9 \\
\hline 4 vocablos & $\begin{array}{l}\text { 1.-Campo } \\
\text { 2.-Flora } \\
\text { 3.-Jardín } \\
\text { 4.-Insecto }\end{array}$ & $\frac{3}{\text { (12 repeticiones) }}$ & $4.28 \%$ & Grupo 10 \\
\hline 3 vocablos & $\begin{array}{l}\text { 1.-Roca } \\
\text { 2.--Desierto } \\
\text { 3.-Hierba }\end{array}$ & $\begin{array}{c}2 \\
\text { (6 repeticiones) }\end{array}$ & $2.14 \%$ & Grupo 11 \\
\hline 7 vocablos & $\begin{array}{l}\text { 1.-Frío } \\
\text { 2.-Arbusto } \\
\text { 3.-Luna } \\
\text { 4.-Ave } \\
\text { 5.-Estrella } \\
\text { 6.-Pez } \\
\text { 7.-Prado }\end{array}$ & $\begin{array}{c}1 \text { aparición en la } \\
\text { muestra }\end{array}$ & $2.5 \%$ & Grupo 12 \\
\hline $\begin{array}{c}280 \text { voca- } \\
\text { blos }\end{array}$ & 38 vocablos & & $99.6 \%$ & \\
\hline
\end{tabular}

De 280 respuestas que comprende el 100\% de la muestra, solamente 38 vocablos son los que conforman la totalidad del centro de interés. Esto refleja un $13.57 \%$. Estas respuestas son los vocablos que representan las entradas léxicas de un diccionario, o sea la unidad léxica. El $86.42 \%$ restante es el porcentaje de repeticiones que aparecieron en el corpus producto de la reiteración en las respuestas.

El grupo número 1 fue quien mayor cantidad de repeticiones tuvo, con 98 repeticiones logró un 35\% distribuido en tan solo 7 vocablos, eso refleja la poca productividad léxica en este centro de interés; con esto se establece que los vocablos: 'agua', 'aire', 'árbol', 'mar', 'flor', 'montaña' y 'río' son los vocablos más genéricos para dicho centro y para todo el estudio de la disponibilidad léxica por lo menos en México $^{16}$. Son las respuestas que una y otra vez aparecerán entre los

16 Esta afirmación se hace porque se ha comparado los resultados de este trabajo con otros proyectos de investigación, los resultados siguen confirmando lo mismo, los vocablos más esenciales para el centro estarán entre las primeras posiciones, en tanto que los vocablos menos genéricos aparecerás en las últimas. 
informantes porque son los objetos más predominantes que pululan al centro de interés "La naturaleza", lo que determinó vocablos muy generales y muy referenciales.

Al aplicar un estudio de la disponibilidad léxica, se obtiene entre los primeros diez lugares los vocablos más disponibles y los más genéricos, conforme se vaya alejando del primer lugar aparecerán los vocablos más complejos para el informante, es decir, las primeras posiciones están condicionadas a las relaciones más referenciales del centro; o sea, a los vocablos con mayor peso significativo y los últimos lugares estarán las especificidades de los vocablos; por ello, vocablos como: 'Frío', 'arbusto', 'luna', 'ave', 'estrella', 'pez' y 'prado', serán las especificidades de este centro de interés. También son considerados como parte de la riqueza léxica de las respuestas de los informantes. Su porcentaje en este análisis comprende $2.5 \%$ con las misma cantidad de apariciones que el primer grupo.

Estos vocablos no son objetos tan sencillos de estratificar en la mente, o sea, son parte del centro pero no son objetos primordiales que deberían estar entre las respuestas más disponibles; por lo que la disponibilidad léxica no sólo refleja la posición de los vocablos, sino también la dependencia referencial en este tipo de estudios.

Entre mayor sea la aparición de un vocablo en un estudio de esta índole, mayor será la estandarización de las respuestas o de los vocablos; o sea, se llenarán las primeras posiciones de vocablos genéricos que establecerán una referencialidad mayor, porque son las respuestas que los hablantes utilizan con más frecuencia, en tanto que entre menor sea su aparición de un vocablo entre los primeros lugares, menor será la referencialidad, será más bien, la puntualidad de la respuesta para ese centro de interés.

De esta forma entre las respuestas del primer grupo con respecto al último, es más usual el vocablo 'agua' que 'arbusto'. El primero es más específico, o sea más referencial mientras que el segundo es más puntual, sigue siendo referencial pero puntual, lo que refleja un mayor grado de dificultad para el informante, puesto que debe sintetizar y recorrer en su léxico mental, información específica con respecto a lo requerido para la muestra.

Estas respuestas condicionarán la distribución de los vocablos en las redes léxicas y en la misma constelación. Su distribución se estable- 
ce en definir: cuáles son los núcleos o bien operadores léxicos de estos vocablos, para ello es importante observar que todos los vocablos de una constelación son operadores léxicos, unos de mayor proficiencia que otros, pero todos engloban en sí, información que se desprende de cualquier vocablo.

\subsection{Análisis Léxico-Semántico}

\subsubsection{Ubicación de los Operadores Léxicos}

Para ubicar los operadores léxicos, primero hay que presentar los vocablos de esta muestra.

Cuadro 2. Vocablos de esta muestra

\begin{tabular}{|l|l|l|l|}
\hline 1.- Agua & 11.-Cielo & 21.-Lago & 31.-Planta \\
\hline 2.- Aire & 12.-Desierto & 22.-Laguna & 32.-Prado \\
\hline 3.-Animal & 13.-Estrella & 23.-Lluvia & 33.-Río \\
\hline 4.-Árbol & 14.-Fauna & 24.-Luna & 34.-Roca \\
\hline 5.-Arbusto & 15.-Flor & 25.-Mar & 35.-Sol \\
\hline 6.-Ave & 16.-Flora & 26.-Montaña & 36.-Tierra \\
\hline 7.-Bosque & 17.-Frío & 27.-Nube & 37.-Viento \\
\hline 8.-Calor & 18.-Hierba & 28.-Pájaro & 38.-Volcán \\
\hline 9.-Campo & 19.-Insecto & 29.-Pasto & \\
\hline 10.-Cerro & 20.- -ardín & 30.-Pez & \\
\hline
\end{tabular}

Tal como se estableció en el análisis estadístico -por lo menos los dos grupos mas representativos, los que denotan la mayor parte de las respuestas cómo el que denota una menor cantidad de las mismas-, los vocablos que se presentan son las totalidades significativas de la muestra. Para establecer cómo se relacionan los vocablos en la disponibilidad léxica, primero hay que definir operador léxico.

Un operador léxico es una serie de unidades mínimas de significado que tienen un carácter, en primera instancia léxico y después semántico ${ }^{17}$. A su vez, estos operadores léxicos forman un conjunto

17 Coseriu, op. cit., p. 79.

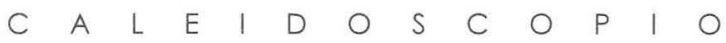


reducido de elementos semánticos que sirven para caracterizar los aspectos sistemáticos y estructurales de un vocablo, con lo que determina piezas léxicas preestablecidas en el discurso lingüístico ${ }^{18}$. El orden de aparición está contactado con rasgos propios del centro de interés, el cual es un especificador para la lengua, o sea, el operador determina las características mínimas de composición que aparecen en los vocablos. Estas características serán que las especifiquen, a su vez, las relaciones entre los vocablos en las redes léxicas.

Un vocablo es un referente del cual pueden emerger nuevos vocablos, Wierzbicka ${ }^{19}$ sostiene que las unidades mínimas de significado son de hecho vocablos de la lengua, los cuales contienen una validez lingüística dentro de la comunidad. Por tal motivo, los vocablos genéricos son los que darán pauta a la expansión de la información.

Un vocablo genérico será, a su vez, un operador léxico. Éste estará enmarcado por el grado de relaciones que se ejecuten y se estratifiquen dentro de la misma red léxica. Se ha enunciado que cualquier vocablo puede ser un operador léxico porque de él surgen nuevas palabras, en esta ocasión definiremos al operador léxico a partir de a) Los resultados de la parte estadística y b) Los argumentos propuestos por Saussure ${ }^{20}$ con respecto a las asociaciones significativas que se dan en el eje paradigmático. Los mismos vocablos -además- son los que determinarán las construcciones o redes léxicas. Se pone como ejemplo el vocablo 'cielo' el cual, de acuerdo a los resultados de los estudios léxicos se ubica este vocablo en el grupo número siete, con seis apariciones, lo que refleja que no es un vocablo tan genérico como se pensara, sino más bien es un vocablo que conjunta información sobre otros referentes, es decir, en su contenido léxico-semántico permite que se incluya en él, otros vocablos importantes por el espacio.

18 Martinet, op. cit, p. 45.

19 Anna Wierzbicka, Lingua Mentalis: The Semantics of Natural Language, New York, Academics Texts, 1980, p. 45.

20 Op. cit., p. 123. 
Las relaciones que se dan entre en el léxico permiten en el hablante desarrollar secuencias de acuerdo al centro de interés ${ }^{21}$, este centro determinará las posiciones en las redes léxicas, o también determinarán relaciones de jerarquía referencial de los vocablos, apoyados por un eje paradigmático; razón por la cual, Saussure ${ }^{22}$ al establecer las relaciones entre asociaciones analógicas y referenciales, se abocaba al estudio de la sistematicidad lingüística; o sea, a los vocablos que dentro de su composición léxica, tienden a generar una mayor relación entre ellos mismos ${ }^{23}$.

Para corroborar esto, véase las relaciones entre los vocablos: 'aire', 'nube', 'lluvia', los cuales parten de 'cielo' por el grado de referencialidad léxica-semántica la cual genera el espacio propicio para involucrar otros vocablos a su relación.

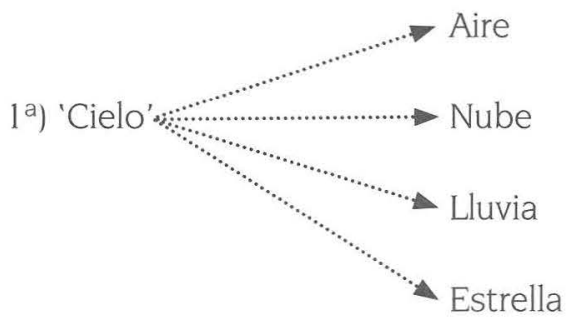

21 Se establece como prioritario para este trabajo de investigación el centro de interés que se basa en el léxico y éste, a su vez, en el vocablo, por otra parte el centro nocional o campo semántico se desarrolla en el léxico-semántico que se encuentra en la lengua.

22 Op. cit.. p. 134.

23 Esto se puede ver en el ejemplo: 'enseñar'. La primera parte abarca relaciones analógicas, o sea, que no tienen ninguna relación con la raíz; en cambio, la derivación morfológica entre 'enseñar'- 'enseñanza' - 'enseño' parte de la misma raíz y denota una mayor profundidad de contenido significativo. 
Obsérvese que no necesariamente un vocablo con un mayor índice de respuesta de acuerdo a la disponibilidad léxica puede ser un operador léxico, en este caso el vocablo 'cielo' funge como tal, es un vocablo que puede incluir en su espacio otro tipo de vocablos, todos ellos relacionados con el operador. Este operador constituye el núcleo de la red léxica. La red es el conjunto de vocablos que se encuentran constituidos por varios operadores. Se rige por un grado de refencialidad específica dentro de la misma composición léxico-semántica ${ }^{24}$. El término operador léxico establece un núcleo preciso que al irse relacionando con otros no pierde su relación, siempre será un operador que pertenecerá a otro núcleo o nodo mayor dentro de la constelación.

De la misma relación entre los vocablos: 'cielo', 'lluvia', 'aire', 'nube' y 'estrella' existen operadores secundarios que son las partes especificativas de las relaciones entre los vocablos, es decir, son todos los vocablos que dependen de esa referencialidad mayor, sin ellos, el o los operadores que se ubican en la constelación no tendrían una relación plausible con la generalidad, por ejemplo, existe una relación de dependencia analógica entre los vocablos 'lluvia' y 'nube'. Esta asociación se enmarca como no necesaria, es decir, hay una relación pero se establece un poco más con relación a la lógica, si hay nubes no necesariamente hay lluvia, lo que indica que dentro de la red existen relaciones secundarias que no alteran el desarrollo conceptual del mismo operador léxico.

La selección de los operadores léxicos forma parte de las relaciones que se generan en la lengua, es decir, al utilizar el discurso lingüístico el hablante selecciona los vocablos de acuerdo a la necesidad comunicativa de cada hablante. Para elegir un operador léxico se establece de la relación de prominencia lingüística que el hablante tiene. Esta elección se enmarca por analogía, o sea todos los vocablos que necesariamente estén inmersos en otro vocablo u otros; por derivación es más complejo el proceso, parte de la morfología, el cual toma como base la raíz y de ella, se empiezan a elaborar relaciones derivativas.

24 Pérez, op. cit, p. 79. 
Se toma como ejemplo la primera relación entre los vocablos:

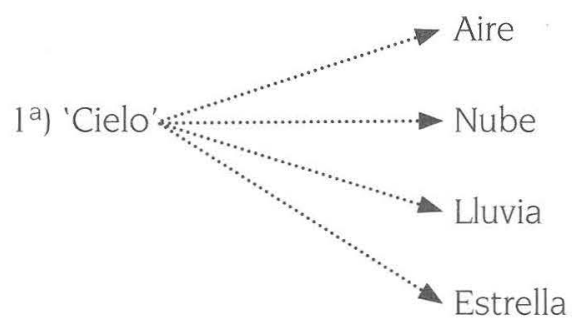

Se ha dicho que un operador léxico es un núcleo dentro de una red léxica, se rige por un grado de referencia específica que abarca una totalidad significativa en la red, repercutiendo en la estructura de la constelación. De manera que todas las asociaciones que se establezcan para cada vocablo están en función del uso de la lengua. Así pues, del vocablo 'aire' -uno de los más disponibles en este centro de interésse puede desprender el vocablo 'pájaro', si se quisiera este vocablo tendría en su estructura otro tipo de información, ya más específica: composición, color, textura, etcétera, que engloban características referenciales de este vocablo.

$1^{\text {b) }}$ 'Aire' 'Pájaro'

También se presenta en todos los vocablos, pero no de manera dispersa sino con relación a los referentes de cada operador léxico ${ }^{25}$, por tal motivo, Saussure ${ }^{26}$ especifica el plano paradigmático para ejemplificar los cambios de significado y de relación por las oposiciones

25 John Lyons, Semántica, Barcelona, Teide, 1977, p. 80.

26 Op. cit., p. 126.

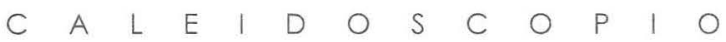


que se manifiestan en el sistema. Es así que miles de entradas pueden ser operadores pero también es obvio que solamente algunas son las que determinarán en la lengua grupos mayores de significación. Los resultados que se dan en la léxico-estadística con respecto a la disponibilidad léxica son única y exclusivamente para constatar, que la riqueza del léxico en los informantes radica en la cantidad de relaciones conceptuadas, entre mayor sea esas relaciones, mayor será la cantidad de vocablos en los resultados de la disponibilidad.

En suma, no existe una riqueza o una pobreza léxica en el discurso, sino más bien, se debe considerar la falta de versatilidad para conceptuar la información y las relaciones entre el operador léxico en el discurso lingüístico. Una red se puede agrandar con base en la inclusión de nuevas entradas, esto significaría generalizar más el operador léxico, entre más general mayor cantidad de vocablos se puede incrustar. La distribución con la inclusión de dos nuevos vocablos a la red permite una mayor generalidad del operador léxico, tal como se mencionó, entre más se generalice mayor será la cantidad de vocablos genéricos que dependan de él; entre más preciso, su referencialidad será específica. Obsérvese el siguiente ejemplo con los vocablos 'sol' y 'calor', que se unen a la primera red ${ }^{27}$.

$2^{\mathrm{a}}$

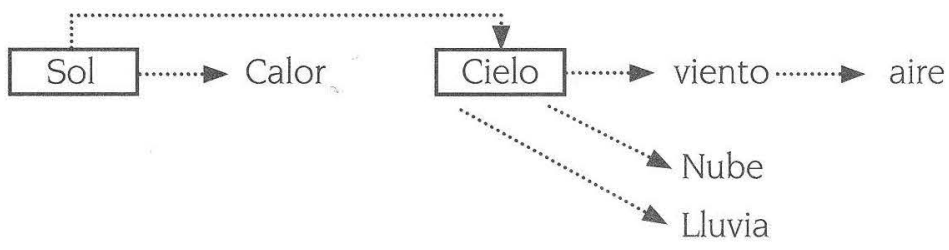

27 Se debe enunciar que estos vocablos pertenecen a los centros de interés "La Naturaleza" que se utilizan en la disponibilidad léxica. Los centros de interés son asociaciones léxicas y léxico-semánticas, las primeras pertenecen a la gramáticas mientras que las segundas a las relaciones conceptuales básicas semánticas. 
Esta red léxica tendrá como núcleos al vocablo 'sol' y al vocablo 'cielo'. Relacionado a 'sol' encontramos al vocablo 'calor', pues aun cuando 'sol' no sea la única fuente de calor, se relaciona que en un día soleado siempre hará 'calor'. En el segundo núcleo, se desarrolla la relación que existe entre 'cielo' y los vocablos: 'aire', 'nube' y 'lluvia'. Así, se nota como éstos mantienen una relación directa con su núcleo.

Continuando con el análisis que se elabora para este trabajo, se enuncian los vocablos: 'flora', 'flor', 'árbol', 'arbusto', 'pasto', 'hierba' y 'planta'. Estos vocablos denotan otro tipo de asociación que se enmarca en una red léxica, la cual da dos tipos de asociación ${ }^{28}$ : analógicas y derivacionales. Las asociaciones analógicas se fundamentan en la relación que se da entre los vocablos, es decir, el operador léxico -'flora'- está marcando un orden que se genera de él. Por derivación, obsérvese estos cuatro vocablos: 'flor' -'flora' y 'árbol' ' 'arbusto'; ambos pares comparten las bases léxicas o bien las raíces léxicas, cuando dos o más vocablos comparten una misma raíz se habla -entonces- de una asociación por derivación. Ésta tendrá entre sus referentes, relaciones morfológicas pero no necesariamente relaciones semánticas. Véase la distribución:

$3^{\mathrm{a})}$

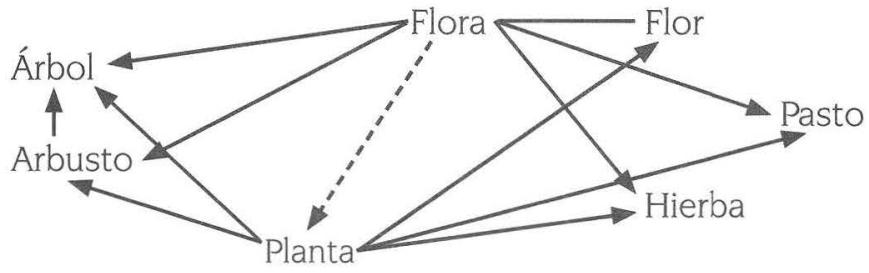

28 Tomando en consideración lo propuesto por Saussure (1916) con respecto a las asociaciones que se pueden dar al momento de elegir el léxico, unas son por analogía, las más comunes, y las otras por derivación que son las más complejas.

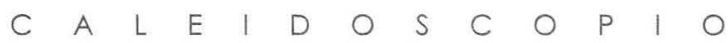


Si se toma el orden de los vocablos en que aparecieron desde la léxico-estadística, se podrá vislumbrar que el vocablo 'flora' se ubicó en el grupo 10, con tan sólo 4.28\%. Esto refleja que la léxico-estadística no permitiría que un vocablo con baja prominencia lingüística apareciera como operador, pero desde la vertiente léxico-semántica, sí se puede.

Aunque la base morfológica sea el vocablo 'flor' permite su estructura tomar como referente el vocablo 'flora', porque es lo universal en la lengua, de esta información se desprende 'flora' y 'fauna' como operadores léxicos universales. Además, 'flora' como tal es un operador porque todos los vocablos que se ubican en su contexto pertenecen indistintamente a él, unos más que otros pero todos llevan consigo información que deban estar unidos a él.

Con lo que respecta a 'árbol'- ' 'arbusto', las similitudes son iguales, el primero es la base y el segundo es la derivación, conforman dentro del sistema lingüístico la creación de una nueva entrada léxica al discurso, 'árbol' apareció en el primer grupo -los más genéricos- mientras que 'arbusto' en el último grupo -los menos genéricos-. Aquí el operador léxico es el más genérico y su derivado forma parte del grupo de este vocablo.

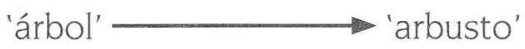

En la red, primero hay una relación con el operador mayor 'Flora' y después con el vocablo genérico 'árbol'.

\subsubsection{Operadores en la Disponibilidad Léxica}

Ya se ha establecido en este trabajo que los operadores léxicos no necesariamente son aquellos con una mayor incidencia léxica, de acuerdo a los resultados de la disponibilidad léxica, puede un vocablo haber presentado una proficiencia lingüística muy elevada pero que no necesariamente funge como operador en la red léxica y en la misma constelación.

Se ha manifestado, también, que un operador léxico lleva consigo información referencial de otros vocablos, funge con un nodo léxico donde converge todo tipo de información dentro del lexicón-léxico 
mental-. Existe una postura al considerar que el léxico se da de manera desordenada y sin ninguna relación en la mente, según ${ }^{29}$ establece que cada palabra que integra el lexicón es considerado como una combinación de tres especificaciones: a) Una morfológica, la cual da la forma de la palabra a la base de temas y afijos; b) Una sintáctica que clasifica la palabra entendiendo a su potencial distribucional en las oraciones y c) Una semántica especificativa que equivale al significado.

De esta manera, el léxico no es una lista desordenada, ni mucho menos caótica, es todo lo contrario, es una lista que se ordena a partir de operadores léxicos bien estructurados. Estos operadores se inician con los resultados de la disponibilidad léxica y fundamentándose en las relaciones léxico-semánticas. Asimismo, el operador que funge como el núcleo de la constelación léxica tiene una aceptación reconocida, aceptación que permite integrar en su base otros vocablos.

El proceso de derivación morfológica se entiende como un proceso que permite ampliar el repertorio de palabras que se originan de un vocablo, para la formación de palabras en la derivación, interviene la misma forma general de reglas léxicas, las cuales enmarcan la unión de un afijo a la base, ya que solamente por este método se puede generar una mayor cantidad de vocablos, formando una tercera palabra ${ }^{30}$.

El proceso de asociación por analogía permite ampliar los grados de referencia entre los vocablos en la red léxica. Por lo que, los vocablos no pueden estar condicionados a un desorden porque necesariamente el mismo vocablo tiende a ordenar sus respuestas, de acuerdo al grado de asociaciones que se generan y que sirven de base para la constelación.

Esta constelación está integrada por las relaciones asociativas analógicas principalmente porque se ubican más en relación al significado que genera el operador léxico. Una red léxica estará en la relación derivacional mientras que una constelación se establecerá en la significación. Obsérvese la composición:

29 Geoffrey Leech, Semántica, Madrid, Alianza Universidad, 1985, p 102.

30 Ibid. 


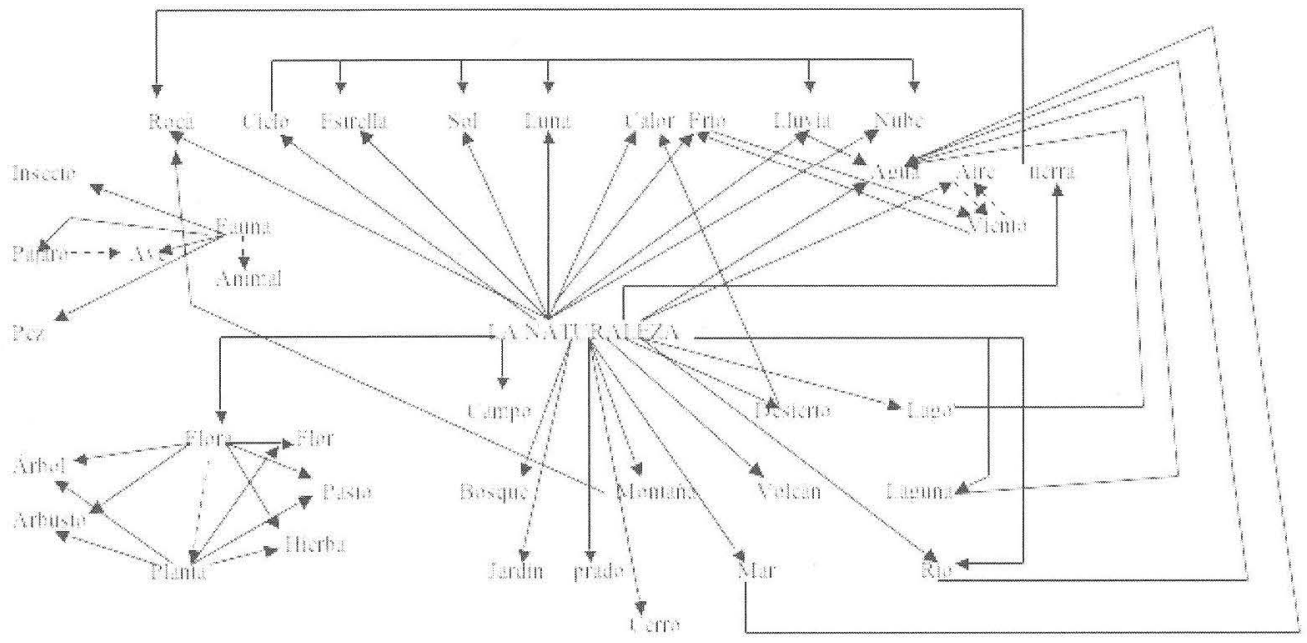

Del anterior constelación se puede constatar que hay varios operadores léxicos, el más grande es el centro de interés "La naturaleza" quien agrupa a todos los elementos por analogía -referencialidad- a su conjunto, las relaciones individuales o precisas estarán establecidas por asociaciones que se dan en el interior de la misma constelación denominadas redes.

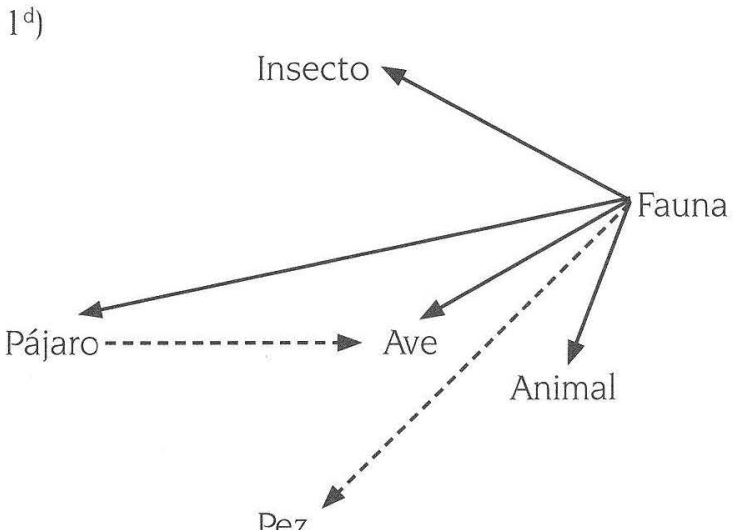


Existen dos operadores léxicos, el primero que conlleva a la unión de todos los vocablos, 'fauna' es el núcleo de esta constelación que dependerá de manera directa de naturaleza, dentro de esta constelación se puede visualizar el vocablo 'ave' como un operador más específico porque de él, se desprende 'pájaro'31 ${ }^{31}$ si se observa es una relación netamente especificativa, luego entonces no se puede hablar más, de que el léxico se encuentra de forma desordenada en la mente, sino más bien que el léxico escoge sus relaciones de referencialidad a partir de sus necesidades comunicativas. El resto de los vocablos 'animal', 'pez' e 'insecto' se dieron como parte de la información perteneciente a la red y a la misma constelación. Si llegara aparecer el nombre de algún animal, entonces el vocablo dejaría de ser parte de la información contextual para convertirse en operador léxico, siempre y cuando se pudiera generar información valiosa dentro de las relaciones léxicas.

En palabras de Leech ${ }^{32}$, es evidente que algunos vocablos se encuentren desactivados en la mente del informante, pero una vez que se activan, generan una bidireccionalidad entre el vocablo y el operador. Esto indica que hay dos sentidos en los que se puede entender un vocablo o una rúbrica léxica: V1: V2; el primero estará sustentado en un aspecto morfológico V1 y el segundo en un aspecto semántico V2.

\section{Conclusiones Parciales}

Lo que se ha expuesto acá es solamente una parte de la verdadera importancia que tiene este estudio en la lingüística, se debe quitar

\footnotetext{
31 Ya se había visto en las anteriores redes que este vocablo puede pertenecer a otro operador como 'cielo', en esta red está más enfocado a la relación de especie y es más especificativo que el anterior operador. Es importante señalar que muchos de los vocablos que pertenecen a los operadores estarán enmarcados primero por las relaciones más específicas, o sea las más necesarias y las más adecuadas, por otra parte esas relaciones, a su vez, establecerán las jerarquías en la constelación, razón por la cual, Saussure hablaba de las relaciones asociativas como fuente para el cambio significativo. Cf. Ferdinand de Saussure, op. cit., pp. 172-177.

32 Op. cit., p. 109.
}

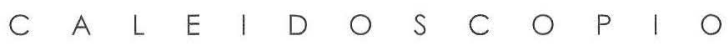


la idea de que el léxico es algo complejo, inestable y sin posibilidad de ordenarse, al parecer eso no es cierto, el léxico es una parte de la lengua que tiene un orden y una especificad en la misma, ¿Cómo se relacionan los vocablos en la disponibilidad léxica?

Los vocablos se ordenan a partir de los datos léxico-estadísticos que se generaron en la recolección de la información; existen, en este marco, vocablos muy disponibles en la lengua que hacen de ellos una reiteración en el discurso. He hablado en otros estudios de la riqueza léxica o bien de la pobreza léxica de los informantes, en ese entonces me basaba en los resultados única y exclusivamente de la disponibilidad léxica, hoy puedo enunciar que no necesariamente los resultados determinan, si existe o no riqueza o pobreza, sino es más bien, las relaciones que se efectúan al interior del léxico del informante.

Estas relaciones se dan a partir de la enunciación o activación de ciertos operadores léxicos que se ubican en la lengua y que determinan enlaces al interior del mismo, Saussure ${ }^{33}$ ya había establecido que estas relaciones se dan en el eje paradigmático, porque solamente ahí es donde se puede generar los cambios de significación en la lengua, pero no es solamente eso, sino también la necesidad de asociarlos a partir de la misma morfología. Ya que esto se establece con relación a la dicotomía entre lengua y habla. Un vocablo se genera al interior del habla mientras que la palabra en la lengua.

Los vocablos se relacionan en la disponibilidad por las asociaciones que se efectúan al interior de la lengua, lo que hace del sistema léxico más estable. Solamente la disponibilidad sirve de base para entender las variaciones del léxico en la lengua y para entender que entre más asociaciones el informante tenga, mayor estabilidad tendrá en su discurso lingüístico. El hecho de que aparezcan vocablos muy genéricos no indicarán que puedan fungir como operador léxicos o bien vocablos regentes

33 Op. cit. 
y regidos. ${ }^{34}$ Si no más bien, es la cantidad de relaciones que de los mismos vocablos emanen dentro de una red ${ }^{35}$, esto no quiere decir que la disponibilidad no tenga un uso en la lingüística, todo lo contrario, el establecer un parámetro en la lengua a partir de la disponibilidad puede llevar a establecer una estandarización del sistema y a comprender los fenómenos lingüísticos de manera más puntual, por ello quien haga un estudio de la disponibilidad léxica estará dando la base para generar estudios más precisos sobre la lengua.

*

34 Esta terminología de vocablos regentes y regidos es dicha por López Chávez, quien establece la necesidad de inclusión de ciertos vocablos a otros, de ahí proviene esta conceptuación. Cf. Iuan López Chávez, op. cit., 2004, pp. 348-360.

35 Se trabaja con redes porque son más estables que las constelaciones, ya que se conceptúan las asociaciones con base en la morfología y la semántica.

C A L E I D 
